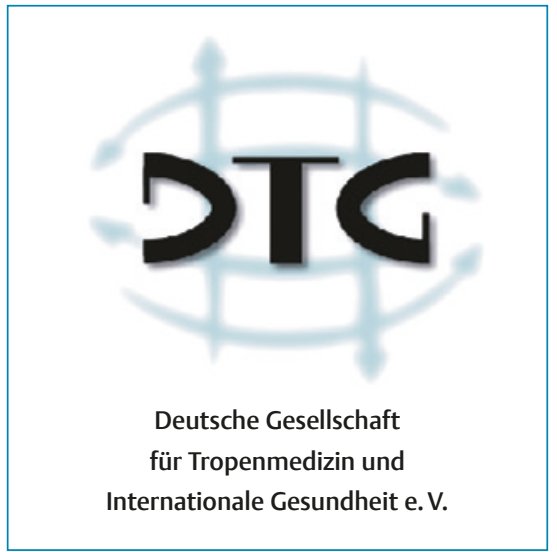

\title{
Liebe Mitglieder und Freunde der DTG,
}

Vom 6. bis zum 10. September fand der European Congress on Tropical Medicine and International Health (ECTMIH) in Basel statt. Die DTG war eine der ausrichtenden Gesellschaften. Vertreter der DTG in den Vorbereitungstreffen war Thomas Junghanss aus Heidelberg.

\section{ECTMIH 2015}

Die Konferenz war hervorragend organisiert und sehr gut besucht. Das Programm war umfangreich mit teilweise bis zu 12 oder 13 Parallelsitzungen, insgesamt wurden etwa 380 Poster präsentiert. Der Schwerpunkt lag - nach unserer persönlichen Einschätzung - auf Public Health, sehr viele Vorträge beschäftigten sich mit dem Thema Point-of-care-Diagnostik von Tropenkrankheiten und natürlich war auch die Malaria mit der Resistenzproblematik und der Entwicklung neuer Medikamente ein Thema in vielen Symposien.

Die DTG hat ein eigenes Symposium veranstaltet, um Schwerpunkte der deut- schen Tropenmedizin bekannt zu machen. Ähnliche Veranstaltungen haben wir schon auf den Kongressen der FESTMIH in Kopenhagen, Barcelona und Verona gemacht. Es wurde daher abgesprochen, dass diesmal die Aktivitäten in Hinblick auf non-communicable diseases darstellt werden. Dieses Symposium war sehr gut besucht und insofern ein großer Erfolg. Folgende Projekte wurde darstellt:

- Torsten Feldt (Universitätsklinik Düsseldorf) stellte Untersuchungen $\mathrm{zu}$ Auswirkungen des Kontakts mit ewaste in Ghana vor. Hintergrund ist, dass jährlich etwa 30 Millionen Tonnen e-waste aus den Industrienationen in LMC exportiert werden - oft illegal. Die Menschen - und das sind oft Kinder - auf den Elektoschotthalden sind zum Beispiel gefährdet beim Verbrennen von Plastik, um Kupfer zu gewinnen.

- Frank Mockenhaupt (Tropeninstitut Berlin) berichtete über Untersuchun-

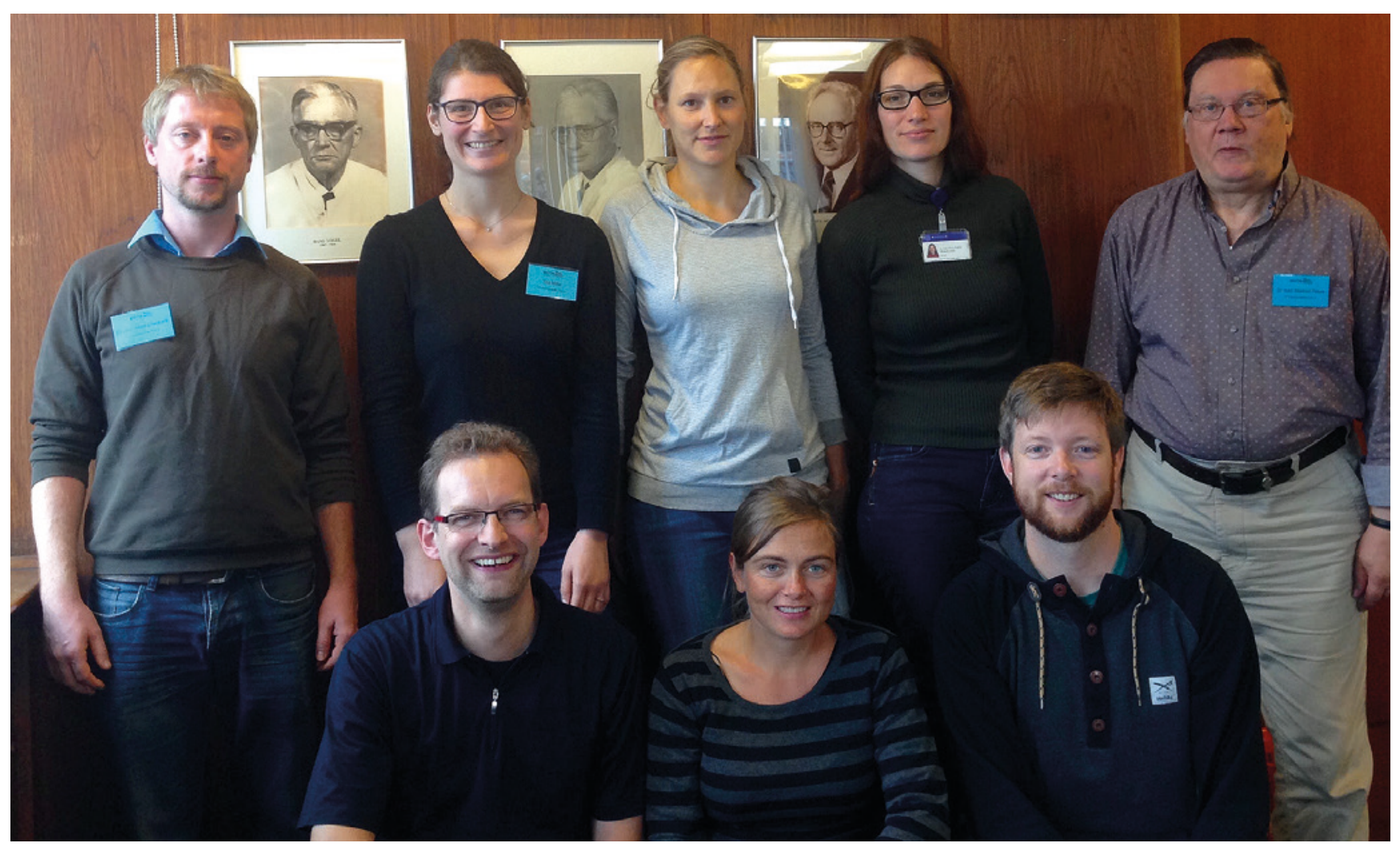

Der Nachwuchsausschuss der DTG tagte zum dritten Mal in 2015. Von links nach rechts, vordere Reihe: Dr. Dr. Carsten Köhler, Ausschussvorsitzender (Tübingen), Dr. Sabine Belard (Berlin), Dr. Benno Kreuels (Hamburg); hintere Reihe: Dipl. Biol. Albert Eisenbarth (Tübingen), Tina Möller (Stuttgart), Dr. Hanna Matthews (Hamburg), Dr. Maria Sophia Mackroth (Hamburg), Dr. Manfred Peters (Hamburg). 
gen zum Diabetes mellitus Typ 2 in Ghana und bei Migranten aus Ghana. Ausgangspunkt ist die steigende Prävalenz des Diabetes. Es wurden aktuelle Ergebnisse der Kumasi Diabetes \& Hypertension Study vorgestellt.

- Ellis Owuso-Dabo vom Kumasi Center for Collaborative Research (Kooperation mit dem Bernhard-Nocht-Institut für Tropenmedizin in Hamburg) stellte weitere Ergebnisse zu Diabetes und Adipositas vor, insbesondere Ergebnisse der RODAM-Studie (Research on Obesity and Diabetes in African Migrants).

- Thomas Junghanss (Sektion Klinische Tropenmedizin, Universitätsklinikum Heidelberg) erläuterte seine Untersuchungen zu den Besonderheiten des Wundmanagements in Afrika - insbesondere unter Beachtung der weiten Differenzialdiagnose bei Hautulzera.

- Günther Fröschl (Tropeninstitut München) stellte die Aktivitäten der Universität München vor, insbesondere das 2009 gegründete Center for International Health at Ludwig-Maximilians-Universität (CIHLMU).

Aus der Vielzahl der anderen Kongressbeiträge und der Symposien sollen noch die beiden Symposien zur Flüchtlingskrise hervorgehoben werden. Es fand eine Plenary Session mit Navid Kermani statt, dem diesjährigen Friedenspreisträger des Deutschen Buchhandels, weiterhin eine Sitzung der European Academic Global Health Alliance (EAGHA) zum Thema „The tragedy in the Mediterranean Sea is a global health issue“, organisiert von Thomas Junghanss und Núria Casamitjana aus Barcelona.

\section{Ausschuss Nachwuchsförderung der DTG}

Im September hat sich bereits zum vierten Mal in den letzten 12 Monaten der Ausschuss Nachwuchsförderung getroffen. Bei dem Treffen in Hamburg wurde, neben einer ersten vorläufigen Auswertung einer Umfrage zum Thema Perspektiven in der Tropenmedizin unter den

Teilnehmern des Diplomkurses Tropenmedizin der vergangenen Jahre, auch die durchgeführte Befragung zur Nachwuchsförderung unter den Weiterbildungsermächtigten in Deutschland analysiert. Dabei zeigt sich, dass in vielen Bundesländern aktuell keine Weiterbildungsermächtigungen in der Tropenmedizin (mehr) vorliegen. Es gilt, Strategien zu entwickeln um dies abzuändern. Der Ausschuss sieht hier unter anderem einen wichtigen Schwerpunkt seiner zukünftigen Arbeit. Junge Mediziner können nur Tropenmediziner werden, wenn genügend Ermächtigte zur Weiterbildung zur Verfügung stehen. Die Ergebnisse der Umfragen sollen nach vollständiger Auswertung veröffentlicht werden. Die Mitglieder des Ausschusses haben zudem zugesagt, die Weiterbildungsermächtigungsseite auf der Homepage der DTG jährlich zu aktualisieren. Zudem soll diese um bereits durch die jeweiligen Landesärztekammern anerkannten Weiterbildungsbescheinigungen ergänzt werden.

Ein weiteres Thema bildete die erste inhaltliche Vorbereitung auf die DTG-Jahrestagung im Oktober 2016. Hier wurden mehrere "Junge-DTG-Veranstaltungen“ zu den Themenbereichen Weiterbildung und Perspektiven in der Tropenmedizin, eine klinisch-tropenmedizinische Fortbildung sowie das Thema Flüchtlingsversorgung angedacht. In diesem Jahr wird im Dezember noch eine Telefonkonferenz des Ausschusses stattfinden. Die nächste persönliche Zusammenkunft ist für Donnerstag, 11. Februar 2016, in Berlin, geplant. Alle Interessierten mögen sich bitte jederzeit an den Ausschussvorsitzenden Dr. Dr. Carsten Köhler (carsten.koehler@ uni-tuebingen.de) wenden.

\section{Aus Hamburg grüßen Sie}

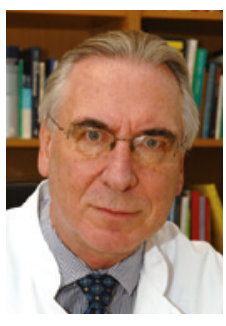

Gerd Burchard

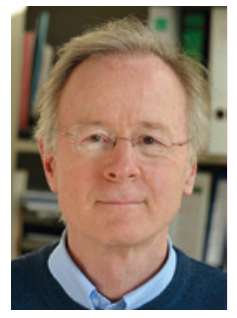

Bernhard Fleischer 